\title{
Annotations
}

\section{Doctors and nurses in neonatal intensive care: towards integration}

Much of the current debate about neonatal intensive care services centres on the broader aspects of organisation at national and regional level. Yet the real organisational challenge lies at the point of delivery of the service-in the neonatal intensive care unit itself-where the demand created by an increasing number of ill preterm babies exceeds the available number of doctors and nurses.

The medical staffing problem at junior level arises from a conflict between the service needs of patients, and the need to protect the career structure of junior doctors. Put simply, if intensive care cots are to be adequately staffed at junior level this would require additional junior staff, in excess of the numbers needed to be trained to fill predicted vacancies for consultant paediatric posts. Implicit in this is the idea that the primary function of junior doctors is to receive training, and that their role in the provision of a service to patients is of secondary importance.

The concept of a neonatal intensive care unit, in which consultants are committed to cot side care, certainly needs to be explored. The nature of neonatal intensive care is such that most consultant paediatricians with responsibilities in this field already spend a substantial proportion of their time directly involved with delivery of the service to the patient in terms of examination, diagnosis, and treatment. An even greater participation in dedicated cot side care, such as carrying out practical procedures, poses the question, 'which other components of the consultant's work might need to be sacrificed?'

Consultants have a continuing responsibility for their patients, a privilege which most of them would be reluctant to forego. Any rota arrangement made with a colleague is for personal convenience, and this temporary cover does not negate a consultant's duty to his or her patient. Thus parents identify the consultant as being ultimately responsible for the care of their baby, and also it is the consultant who is in a good position to discuss with parents in a consistent way the varied and often complex aspects of their baby's present and future health. In most cases continuity extends to the outpatient clinic after the baby is discharged. A radical change in the work pattern of consultants on neonatal intensive care units would have to be based on a formal rota or shift system-at the expense of this continuity of personal care and responsibility. This cannot be in the best interests of patients and their parents, nor is it the most cost effective use of a consultant who spends a minimum of 15 years in undergraduate and postgraduate training before being appointed.

The basis of neonatal nursing staff shortages is different. Some neonatal intensive care units do not even have a numerically defined establishment of nursing staff. Financial restraint does play an important part in limiting the numbers of neonatal nurses but it is not the only problem. Many neonatal intensive care units, even some traditionally prestigious ones, are experiencing problems in recruiting nurses, and many nurses leave within a short time of being appointed. Poor job satisfaction, lack of a creative role in their work, physical and emotional pressures of neonatal intensive care, and poor salary all contribute to this. Moreover, there is a shortage of trained neonatal nurses from which to draw (see page 760).

We have reached a cross road with respect to staffing of neonatal intensive care units, and perhaps now is the time to reflect whether traditional work patterns could be modified. The nature of the clinical work on neonatal intensive care units falls into different but overlapping categories: diagnosis and treatments including the prescription of drugs; monitoring and observations; responding to emergencies; practical procedures; conventional nursing hygiene care; and attending to the very legitimate needs of parents.

Can we conceive a strategy that would permit a better integration of nursing and medical care for the benefit of babies, and which would at the same time make the work of neonatal nurses more creative, and perhaps encourage recruitment to the specialty? There is a danger in viewing this solely in terms of an extended role towards carrying out practical procedures, currently the responsibility of doctors. Although this might satisfy some nurses who are gifted in this field, it might tend to encourage the separate growth of nurse technicians and 'task orientation', which creates further polari- 
sation and is contrary to the principle of integration.

A key factor which distinguishes the work pattern of the nurse and the doctor at the point of delivery of the service is that the nurse is in constant contact with the baby when she is exposed to rapidly evolving clinical situations. The late recognition of warning signs in babies can lead to a relentless cascade of disturbed physiology, culminating in a downward spiral of clinical deterioration, with potential long term neurodevelopmental and other health implications for surviving babies. Neonatal nurses already use their skills to recognise deviations from the norm in respect of the observations they make. Yet there is a paradox, in as much as the growth of electronic monitoring of vital signs and blood gases carries the risk of divorcing nurses (and doctors) from the totality of a baby's medical needs. Indeed, nurses in some busy units already comment that under the pressure of staff shortages "hourly observations' devolve into a boring ritual of transferring electronic readings into the nursing records. What is recorded on the chart might not give any 'feel' about the baby's true progress.

The fact of staff shortages is so entrenched that it could habitually dictate an unfavourable work style for neonatal nurses for the future, thus continuing the cycle of poor incentive and poor recruitment. That is why we should not let current staff shortages deter us from exploring the idea of better integration of the skills of the nurse and doctor. The cot side devotion of neonatal nurses provides an opportunity for them to extend their clinical skills as diagnosticians-inferring from events and clinical assessment the nature or cause of particular situations-before the baby enters that downward spiral of deterioration. Against this solid background those who wished to could, with appropriate training, build on this and carry out procedures such as putting babies on ventilators, sampling and measuring arterial blood gases, adjusting ventilator settings, inserting peripheral lines and arterial catheters, draining pneumothoraces, and treating hypovolaemic shock.

Even where a regional neonatal intensive care referral service operates, the district special care baby units cannot ignore their responsibilities for their sick babies, nor would they wish to. These units are increasingly called on to provide a service, including monitoring of unstable babies and vigilance for complications, which is beyond that enshrined in the expression 'special care'. Many of these units also provide mechanical ventilation, sometimes beyond 24 hours, until a place can be found for a baby in a referral unit. This situation will not go away; nor is it likely that the present constraints on the creation of new posts at senior house officer and registrar grades will be lifted to meet the needs of these babies, who, in many cases, are not receiving the care they deserve. The provision of appropriately trained neonatal nurses in sufficient numbers working with the registrar and consultants in district special care baby units would help to alleviate this problem. There is no reason why the job content of junior medical staff would be weakened; on the contrary, it would certainly enhance training opportunities for senior house officers in district hospitals. In the regional centres they would still retain a large role in neonatal care, only sharing their responsibilities with neonatal nurses.

It is appreciated that not all nurses working in neonatal intensive care have long term career motivations in this specialty. Those who do would, after training, contribute to a core of such nurses in district hospitals and regional centres. Thus integration would improve services for the newborn and at the same time provide a real opportunity for certain nurses to pursue a career in neonatal medicine in a way that promoted job satisfaction. It is essential that nurses and doctors each know where their responsibilities lie because this helps to secure the safety of patients. An integrated work pattern raises the issue of the extent to which neonatal nurses can be regarded as autonomous decision makers. A major change would be required to change the fundamental precepts that govern the regulations for the prescription of drugs. The wellbeing of ill newborn babies, however, depends far more on factors other than the medicines they are prescribed.

Those debating autonomy should not be shy of allowing undercurrents to surface, because it is only by frank discussion that the nursing and medical professions learn to respect each other's anxieties. Doctors do pose a threat to some nurses. It is easy to understand the potential conflicts which exist at present when experienced neonatal nurses work alongside less experienced senior house officers, who rotate through the unit every six months or less, and who, none the less, are responsible for prescribing a wide range of treatments, albeit under consultant supervision. The fact that each patient is admitted under the care of a named consultant who is responsible for directing treatment might also be a source of intimidation for some nurses, but the extent to which this occurs usually reflects the personality and approach of the consultant.

Integration is not about the right to prescribe treatments and the right to be in charge of patients. Instead it is about the degree of responsibility nurses wish to accept and doctors are willing to share. Those who have been appropriately educated and 
trained and who have proved themselves under supervision, be they doctors or nurses, have earned the right to perform whatever they have been taught to do. None of us is autonomous when it comes to looking after patients, and much can be done to resolve potential conflicts between nurse and doctor in an integrated service. Indeed, forward looking units already operate within a framework that encourages good working relationships.

Firstly, each unit should have a policy which serves as a broad guide for the management of babies with various disorders. This policy, decided jointly by medical and nursing staff, should combine elements of traditional nursing care with medical treatment. It should be the subject of regular formal reviews and revision to take account the pace of change occurring in neonatal practice.

Secondly, it is now common for individual nurses on each shift to identify with particular babies to provide an element of continuity of care throughout the shift; in addition, several experienced neonatal nurses, drawn from the core who have received special training, should work outside the traditional shift system and they, together with medical staff, should provide continuity of care between shifts.

Thirdly, by using their extended clinical skills continuously by the cot side the contribution of nurses to ward rounds is considerably enhanced. Under these circumstances joint ward rounds permit more critical planning of a baby's total needs and are intellectually stimulating and educational for all the staff.

Fourthly, it might be helpful to have joint medical and nursing representation (at an appropriate level) on interview committees for staff appointments to neonatal intensive care units. There is scope for assessing more critically a candidate's potential for work in neonatal intensive care because a nurse or doctor might appear superficially to be suited to an integrated work arrangement and yet might prove to be a burden.

Integration cannot be considered without first giving detailed attention to the numbers of nurses required to work such a system and the appropriate mix of learners and experienced staff. This will entail research into work load in relation to work patterns, and a review of current nursing and medical procedures because some routines may have developed by habit and are neither efficient, nor of benefit to the baby.

Although it is essential for neonatal units to have their own 'establishment' of nurses, staff levels should emphasise the number of experienced nurses and learners required for duty on each shift in relation to work load at that time. One of the serious management problems posed by neonatal intensive care is the erratic fluctuation in work load. It is unfortunate that at present trained and experienced neonatal nurses who offer themselves for part time work are not always welcomed because of inflexible local policy governing the employment of part timers.

The education and training requirements to meet an integrated service need to be addressed by the appropriate professional nursing organisations. Attendance at a prescribed course of study, such as one of the current English National Board courses on neonatal intensive care, is certainly not the sole requirement. There is, even now, an urgent need to develop a form system of in-service training for neonatal nurses, emphasising cot side teaching. Intensive care units require the services of neonatal nurse teachers, and these posts should be seen as prestigious senior positions. The present avenues for promotion in nursing care entail ascending the ladder away from patients, and there is a need to retain trained and experienced sisters where the service is delivered. Their service input to patients should allow them adequate time to give learners the benefit of their experience, and this should be seen as their primary role. These teaching posts could also be used for training midwives on postnatal wards, thus facilitating the practice of special care on the wards and avoiding unnecessary admission of babies to the neonatal medical unit.

Integration must be seen for what it really isnamely, as a genuine desire to bring about better care for sick babies and better job satisfaction for nurses, against the backgrounds of what is potentially a very serious crisis in medical staffing, which other specialties will also have to come to terms with in their own way. It cannot be contemplated without a review of nurse staffing requirements because it would be a retrograde step if it developed at the expense of the many other facets of care that nurses currently provide, including total family care and comforting grieving parents. One attraction of integration is that it provides the proper framework for neonatal nurses to assume a more responsible role, not only with parents, but with key professional groups working in neonatal services, such as social workers, and it also opens up realistic avenues for research by neonatal nurses. The hallmark of innovative management is not to despair in the face of a crisis but to exploit the situation to bring about change.

We are very grateful to Angela Carton, Clinical Nurse Manager, Neonatal Medical Unit, North Western Regional Perinatal Centre, St Mary's Hospital, Manchester, for her helpful comments.
M L Chiswick
Manchester
N C Roberton Cambridge 